\title{
Drug-related problems identified by clinical pharmacists in nephrology department of a tertiary hospital in China-a single center study
}

\author{
Xing-Xing Liu ${ }^{1,2 \#}$, Hui-Xia Wang ${ }^{3 \#}$, Yun-Yun Hu${ }^{4 \#}$, Xu-Ting Zhu ${ }^{5}$, Xin Tan ${ }^{6}$, Yang Yang ${ }^{7}$, Yong-Fu Hang ${ }^{1}$, \\ Jian-Guo $\mathrm{Zhu}^{1}$ \\ ${ }^{1}$ Department of Pharmacy, the First Affiliated Hospital of Soochow University, Suzhou, China; ${ }^{2}$ Department of Pharmacy, Guiyang Maternal and \\ Child Health Care Hospital, Guiyang, China; ${ }^{3}$ The People's Hospital of Danyang, Affiliated Danyang Hospital of Nantong University, Danyang, \\ China; ${ }^{4}$ Department of Pharmacy, Suzhou Xiangcheng People's Hospital, Suzhou, China; ${ }^{5}$ The Affiliated Hospital of Jiangnan University, Wuxi, \\ China; ${ }^{6}$ Northern Jiangsu People's Hospital, Yangzhou, China; ${ }^{7}$ Wuxi No.2 People's Hospital, Wuxi, China \\ Contributions: (I) Conception and design: YF Hang; (II) Administrative support: JG Zhu; (III) Provision of study materials or patients: All authors; \\ (IV) Collection and assembly of data: HX Wang, YY Hu, XT Zhu, X Tan, Y Yang; (V) Data analysis and interpretation: XX Liu, HX Wang; (VI) \\ Manuscript writing: All authors; (VII) Final approval of manuscript: All authors. \\ \#These authors contributed equally to this work. \\ Correspondence to: Yong-Fu Hang; Jian-Guo Zhu. Department of Pharmacy, the First Affiliated Hospital of Soochow University, Suzhou 215006, \\ China. Email: hangyongfu1986@163.com; 15950005195@163.com.
}

\begin{abstract}
Background: There is a lack of data on drug-related problems (DRPs) occurring in nephrology department in China. The objective of this study was to identify and categorize the types and causes of DRPs and to assess their severity. DRPs were examined by clinical pharmacists and the results of their interventions were rated.

Methods: Clinical pharmacists reviewed all medication orders for patients and documented clinical pharmacy services within a nine-month study period. The Pharmaceutical Care Network Europe (PCNE) classification (Version 9.00) was used to identify DRPs. Our Primary outcomes measured the number, causes, types, potential hazards of DRPs and the types and success rate of intervention.

Results: Admission medication reconciliation data of 113 patients with chronic kidney disease (CKD) were collected and all of the medications were reviewed retrospectively. Exclude 26 patients who did not occurred DRPs, 87 patients (77\%) identified 101 DRPs. The average DRP number per patient was 1.16. The most common type of problem was "treatment effectiveness P1" $(84.16 \% ; 85 / 101)$. The most common causes were "drug selection C1" (36.00\%; 45/125), "dose selection C3" (29.60\%; 37/125), and "patient related C7" (26.40\%; 33/125). Clinical pharmacists totally proposed 249 interventions, of which 190 (76.31\%) were fully accepted and implemented.
\end{abstract}

Conclusions: DRPs are common among CKD patients in the nephrology department. Hence the necessity for pharmaceutical care to be improved to ensure the ongoing safety of patients.

Keywords! Drug-related problems (DRPs); chronic kidney disease; clinical pharmacist

Submitted Mar 15, 2021. Accepted for publication Aug 17, 2021.

doi: 10.21037/apm-21-817

View this article at: https://dx.doi.org/10.21037/apm-21-817

\section{Introduction}

Chronic kidney disease (CKD) is an important health problem. its global prevalence was estimated at $9.1 \%$ in
2017 (1). It has been considered that on the list of the leading causes of early death, CKD will rise from 16 th to 5 th position by 2040 (2). Globally, China is the most 
populous developing country and the prevalence of CKD was reported to be $10.8 \%$ (3). Unfortunately, a formal CKD control system has not been established. In addition, management and intervention measures were not carried out in a timely and effective manner, which has in turn led to a significant increase in the prevalence of end-stage renal disease in China and a huge consumption of medical resources (4).

Drug-related problems (DRPs) include identification, prevention, and solution. They are the core processes of pharmaceutical care. In accordance with Pharmaceutical Care Network of Europe (PCNE), a DRP is defined as, "an event or circumstance involving drug therapy that actually or potentially interferes with desired health outcomes" (5). Through medical workers prevent actual and potential DRP, to ensure patients safety and effectiveness of the treatment effect. It is very important for DRPs to be documented in the pharmaceutical care process (6) and while there are several classifications (7), no single standardized classification for DRPs exists. Comparing with other classifications, the PCNE classification system is more often practiced and has better usability and internal consistency than others as it is updated and revised periodically.

As a chronic disease, there are many factors influencing the treatment effect of CKD.

The characteristics of CKD include two points. One is the changes of drug pharmacokinetics due to the disease itself, another is the use of multidrug therapy and reactive immunosuppressive drugs. Therefore, the phenomenon of DRP in CKD patients is very common and specialized personnel are required to fully supervise and monitor patients' medications. In addition, the effective concentration range of immunosuppressive drugs is narrow. Such drugs include tacrolimus and cyclosporine. Serum concentration monitoring is needed in both of these drugs. In short, DRP management is very important (8).

The clinical pharmacists' basic working principles are to provide pharmaceutical care. Pharmacists have the expertise to detect, solve, and prevent medication errors and can help clinicians find DRPs. There are many things that clinical pharmacists can do that are beneficial to clinical treatment, including optimize drug treatment, adjust drug dosage, conduct medication reconciliation, monitor laboratory indicators and improve patients' medication compliance through educate patients on medications. Unquestionably, therefore, pharmacists are indispensable members of a complete treatment team (9). In addition to DRPs, many factors affect the therapeutic effect of CKD, such as medication compliance, life style of patient, severity of the disease and so on.

There are many factors that can increase prevalence of end-stage kidney disease in China, but we lack of data related to drug-related problems (DRPs). There is limited information on interventions by clinical pharmacists among CKD patients in Chinese hospitals. Using the PCNE classification system, we investigated the incidence and nature of DRPs in a nephrology department in a hospital in Suzhou. The Morisky Medication Adherence Scale (MMAS-8) (10) is the subjective measures and it use to assess medication adherence in chronic diseases. It is a patient self-reported tool which transform 8 items related to medication taking behaviors into an adherence score. In our study, we used the MMAS-8 to assess medication adherence of CKD patients.

We present the following article in accordance with the STROBE reporting checklist (available at https://dx.doi. org/10.21037/apm-21-817).

\section{Methods}

\section{Study design and patients}

This study was carried out in a tertiary hospital in China from December 2017 to September 2018. The inclusion criteria included: (I) patients aged $\geq 18$ years and diagnosed with CKD; (II) the numbers of drugs used before admission was $\geq 1$. The exclusion criteria included: (I) the unavailability of a complete medication history; (II) the inability of the clinical pharmacists of patients to communicate effectively within $24 \mathrm{~h}$ due to various reasons; (III) the unwillingness of patients to cooperate with clinical pharmacists in medication reconciliation work. All procedures performed in this study involving human participants were in accordance with the Declaration of Helsinki (as revised in 2013). The study was approved by the Ethics Committee of the First Affiliated Hospital of Soochow University (NO.: (2021)146) and informed consent was taken from all the patients.

All data were performed by the clinical pharmacists of the Department of Nephrology face to face. The information was collected for each patient includes age, gender, body height and weight, admission diagnoses, previous medical history. By asking the patients, the clinical pharmacists compiled the pre-admission medication and admission medication lists. Finally, patients received a comprehensive assessment for medication adherence by MMAS- 8 . The impact of interventions was assessed by: (I) the potential severity of DRPs by Bayliff (11); (II) the number of DRPs 
Table 1 Types of DRPs as identified by the PCNE DRP classification tool V9.00

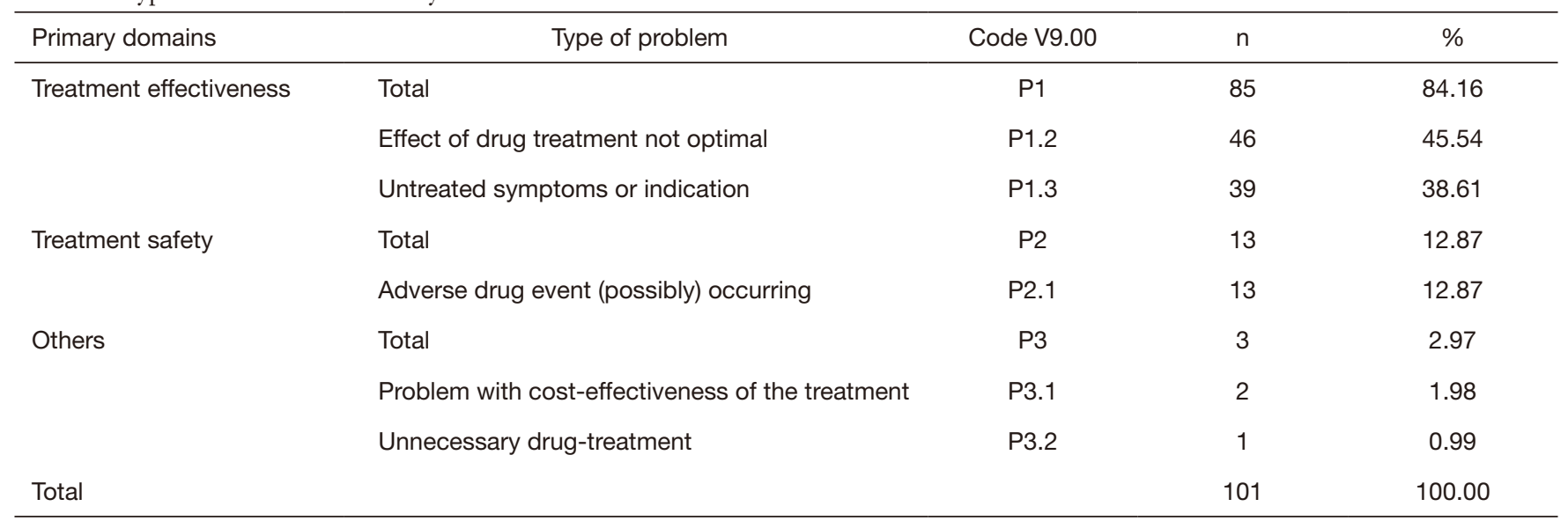

DRP, drug-related problem; PCNE, Pharmaceutical Care Network Europe.

and acceptance of intervention.

The PCNE classification (Version 9.00), which was last updated in February 2019 (5), was used to evaluate the DRPs. All DRPs in nephrology patients were documented, collated, and entered into a data sheet. We used PCNE classification to identify DRPs by five primary domains. For instance, problems, causes, interventions, and acceptance of the intervention proposals. The five sections were further divided into 23 main domains, and then into 78 grouped subdomains.

Furthermore, the severity rating of DRPs were evaluated using the classification system devised by Bayliff. This classification consists of four categories based on the severity of the outcome: (I) no effect on clinical outcomes (category 0); (II) potentially mild clinical effects (category 1); (III) outcome caused treatment or prolonged hospitalization (category 2); (IV) outcome endangered life (category 3). The total scores on the MMAS- 8 range from 0 to 8 , and are categorized into three levels of adherence: high adherence (score $=8$ ), medium adherence (score of 6 to $<8$ ), and low adherence $($ score $<6)$.

\section{Outcomes and data analysis}

According to the PCNE classification (Version 9.00), We collected the types and causes of DRPs, and made statistics on the results of intervention and acceptance of recommendations. On top of that, categorical variables were expressed as numbers and percentages, continuous variables were expressed as mean with standard deviation.

\section{Results}

Of the 113 patients taking medication for CKD, 87 patients had DRPs (77\%). These include 40 females and 47 males with a mean age of $48.59 \pm 15.12$ years old, including 9 elderly patients (10.34\%, more than 65 years old). In the light of the estimated glomerular filtration rate (eGFR), 27 patients $(31.03 \%)$ were CKD stage 1 or 2,14 patients (16.09\%) were CKD stage 3, 12 patients (13.79\%) were CKD stage 4, and 34 patients (39.08\%) were CKD stage 5.

\section{Assess Medication Adberence}

Among the 87 patients, based on the MMAS-8, 12 patients $(13.79 \%)$ had high adherence scores (score $=8), 34$ patients $(39.08 \%)$ had medium adherence scores (score of 6 to $<8$ ), and 41 patients $(47.13 \%)$ had low adherence scores (score $<6)$. The average score of adherence was $5.7 \pm 1.83$. In total, $86.21 \%$ of the patients could not fully comply with the doctor's instructions when taking drugs.

\section{Identifying DRPs}

As shown in Table 1, the 87 patients had 101 DRPs, which were classified by the PCNE V9.00 as an average of 1.16 per patient. The results showed that there were 85 problems of "treatment effectiveness P1" (84.16\%; 85/101), 13 of "treatment safety P2" (12.87\%; 13/101), and 3 classified as "others P3" (2.97\%; 3/101). Detailed information is shown in Table 1. 
Table 2 Causes of DRPs were identified by the PCNE DRP classification tool V9.00

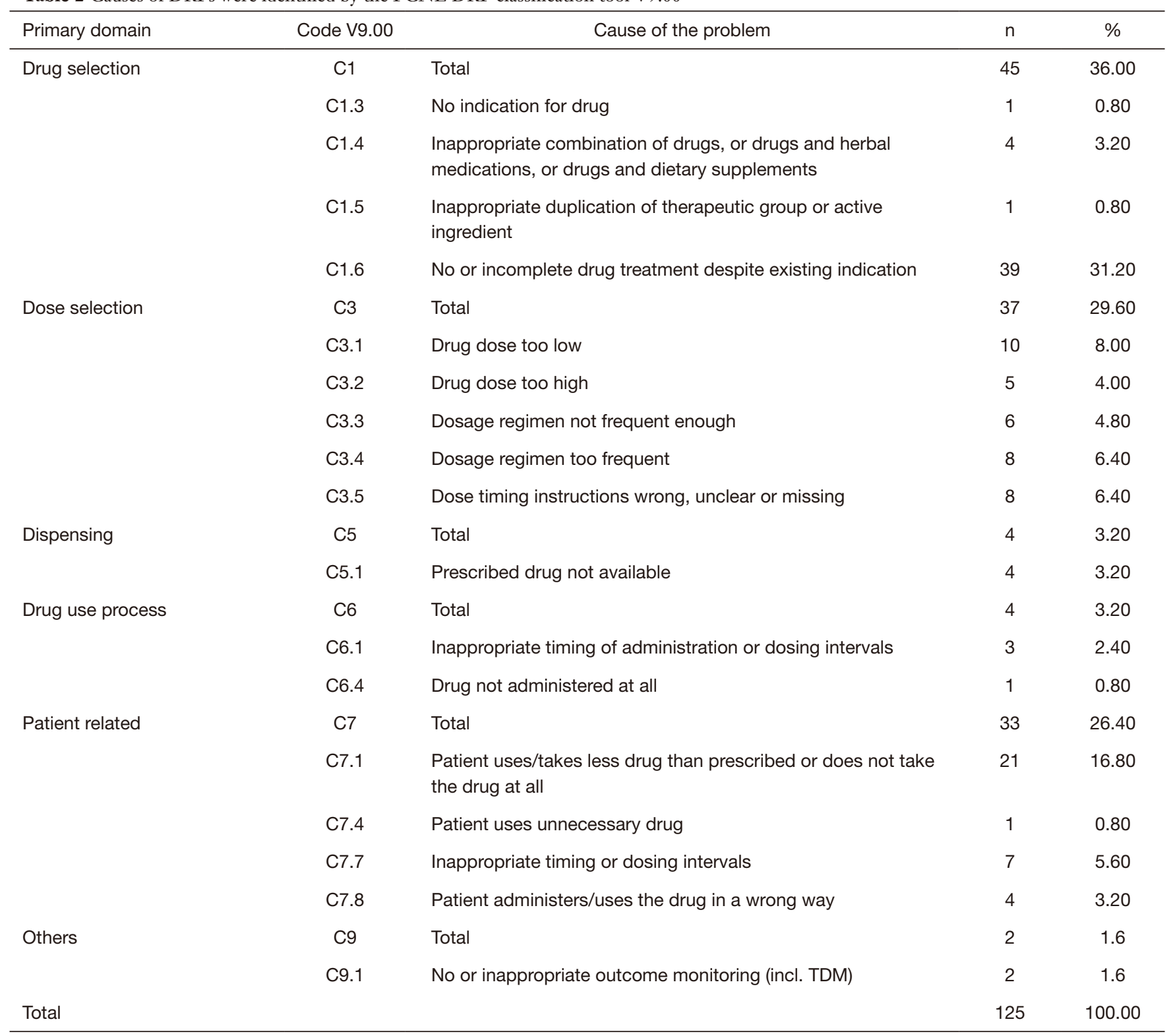

DRP, drug-related problem; PCNE, Pharmaceutical Care Network Europe.

\section{Identified causes of DRPs}

As we can see from Table 2, clinical pharmacists found a total of 125 reasons for the 101 DRPs. The most common causes were "drug selection C1" (36.00\%; 45/125), "dose selection C3" (29.60\%; 37/125), and "patient related C7" (26.40\%; 33/125).

The Bayliff tool was used to evaluate the potential hazards of the 101 DRPs. The results showed that there were 3 (2.97\%) grade category 0 hazards, 85 (84.16\%) grade category $1,13(12.87 \%)$ grade category 2 , and no category 3 hazards, as shown in Table 3.

After identifying the 125 reasons for DRPs, clinical pharmacists proposed 249 interventions, of which 190 (76.31\%) were fully accepted and implemented. Among them, 96 reasons for the DRPs were placed "at the prescriber level I1" with 61 reasons for intervention and full implementation, and the success rate of the intervention was $63.54 \%$. A further 75 reasons for the DRPs were placed "at the patient level I2", with 67 reasons for 
Table 3 Potential risk of DRPs of DRPs

\begin{tabular}{lcccc}
\hline & \multicolumn{2}{c}{ Drug-related problem $(\mathrm{n})$} & \multicolumn{2}{c}{ Total } \\
\cline { 2 - 4 } Damage rating & CKD1-2 & CKD3-5 & 3 & 2.97 \\
\hline Category 0 & 1 & 2 & 85 & 84.16 \\
Category 1 & 23 & 62 & 13 & 12.87 \\
Category 2 & 2 & 11 & 0 & 0.00 \\
Category 3 & 0 & 0 & 101 & 100.00 \\
Total & & &
\end{tabular}

DRP, drug-related problem; CKD, chronic kidney disease.

Table 4 Types of intervention, its acceptance and implementation as identified by the PCNE DRP classification tool V9.00

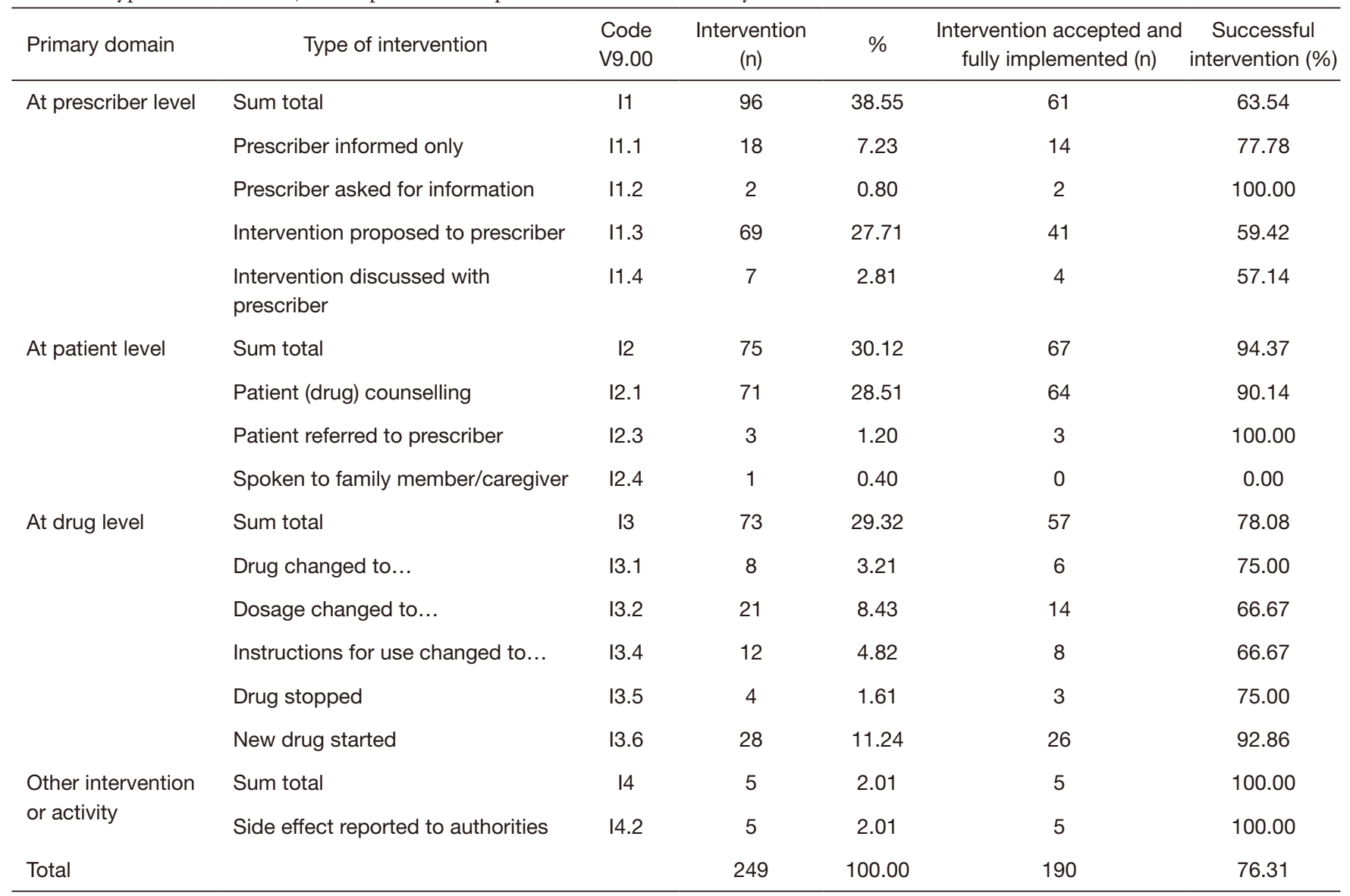

DRP, drug-related problem; PCNE, Pharmaceutical Care Network Europe.

intervention and full implementation, and the success rate of intervention was $94.37 \%$. There were 73 reasons for the DRPs placed "at the drug level I3", with 57 reasons for intervention and full implementation, and the success rate of intervention was $78.08 \%$. Finally, there were five reasons for "other intervention or activity I4" which saw five interventions fully implemented, and a success rate of the intervention was $100 \%$. Table 4 depicts all data on the types of intervention and its acceptance and implementation.

DRP status reflects the results of the intervention. Finally, 69 out of 101 DRPs $(68.32 \%)$ were completely solved, $14(13.86 \%)$ were partially solved, 17 (16.83\%) 
Table 5 Outcomes of intervention were identified by the PCNE DRP classification tool V9.00

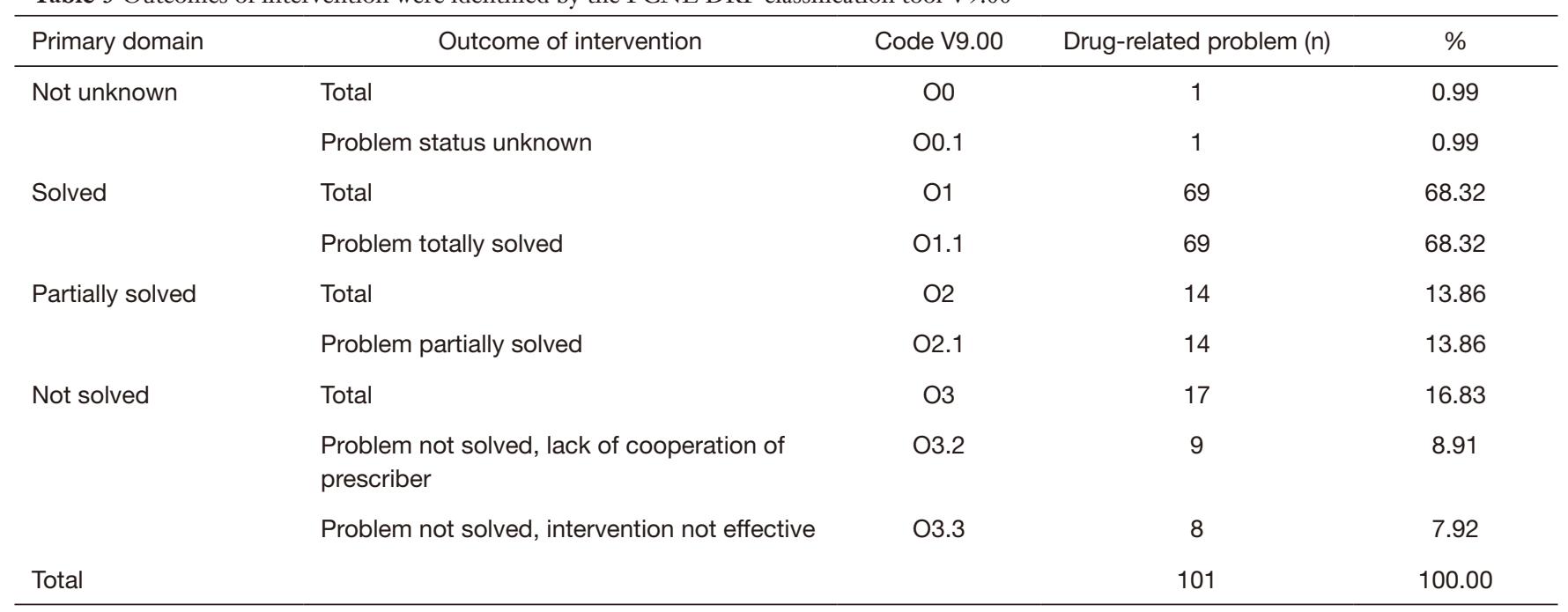

DRP, drug-related problem; PCNE, Pharmaceutical Care Network Europe.

were unresolved, and $1(0.99 \%)$ was in an unknown state, as shown in Table 5.

\section{Discussion}

Our study is the first report on DRPs in a nephrology department using the PCNE classification tool V9.00 in China and the results showed that the 87 patients involved had 101 DRPs (an average of 1.16 DRP per patient). The mean age of the study population was $48.59 \pm 15.12$ years old, which was close to the mean age found in the study conducted by Subeesh et al. (12), reflecting the fact that there is a high prevalence of CKD among people with relatively high age.

In our study, the major types of DRPs were "treatment effectiveness" and "treatment safety", which accounted for over 97\% (98 of 101 DRPs), and the most prominent problem was "effect of drug treatment not optimal", which accounted for $45.54 \%$ of the problems (46 of 101 DRPs).

Within the causes of DRPs, "drug selection" and "dose selection" were the major aspects, accounting for more than $65.60 \%$. While "no or incomplete drug treatment despite existing indication" was the major cause in the "drug selection" domain. Within the "dose selection" domain, "drug dose too low", "dosage regimen too frequent", and "dose timing instructions wrong, unclear or missing" accounted for more than half of the causes. Dvořáčková et al also conducted a study showed that the most frequent DRPs were untreated indication (27.18\%) and incorrect dose, too low $(20.81 \%)$ which was similar with ours (8). The results showed that the intervention of pharmacists in clinical medication management was very important. Specialists may only focus on drug treatment in undergraduate departments, while other problems experienced by patients are often easily overlooked. Consequently, this result reminds us that pharmacists should strengthen the medical order examination to ensure the safety of medication and achieve the goal of treatment. Moreover, as the kidney disease worsening, more and more medications are used to treat related complications. Such as anemia, mineral, bone disorders and so on. The CKD patients who reach endstage renal disease, they may need take 10-12 drugs (13). Due to CKD patients need long-term integrated treatment and repeated admission and discharge, doctors may forget to prescribe drugs or mistakenly provide incomplete drug treatment. Clinical pharmacists can identify these DRPs and prevent potential DRPs. This provides a guarantee for the safe and effective treatment of patients (14).

The potential severity of DRPs was rated by the Bayliff tool. Category 0 means it has no impact on clinical outcomes, category 1 means there is potentially mild clinical effect, category 2 means there is a potential clinical impact that would trigger the need for treatment or extended hospital stay, and category 3 means the effect might be fatal. Through our research, the more severe the disease was, the more diseases were combined, and the more drugs were used. We found that as the severity of CKD increases, the frequency of DRPs also increases, but the relevant 
information needs to be verified in a larger sample. In our study, we did not encounter life-threatening circumstances in patients with drug-related problems. This, however, could be caused by the sample size.

In our study, there were 85 DRPs rated at category 1 and clinical pharmacists proposed interventions after identifying the DRPs. Most of the interventions were fully accepted and implemented (190/249) and the prescriber acceptance rate was high "at patient level" (94.37\%). From the other 59 interventions which were not being accepted and implemented, 50 interventions were accepted but partially implemented, 4 interventions were accepted but implementation was unknown, and 5 interventions were not accepted because the doctors did not reach consent. Analyzing the reasons, we can see that the acceptance rate of doctors is relatively high in the following two conditions: not giving patients treatment of drugs while symptoms exist, and unnecessary or surplus use of drugs. By comparison, the acceptance rate of doctors is low in these two conditions: inaccurate drug doses and inaccurate timing of administration of drugs. Some doctors will ignore the impact of adequate drug use on the effect of treatment. Nonetheless, medication compliance of patients in our study was not satisfactory. Among 87 patients, only 12 (13.79\%) had high medication adherence. According to our study, there are many reasons which can lead to poor medication adherence in CKD patients, such as patients' age, patients' education background, patients' economic income, patients' anxiety about the side effects of drugs, the fact that patients do not pay enough attention to their own diseases, the fact that patients wouldn't completely trust the doctors, and so on. Through the active intervention of clinical pharmacists, the medical education of CKD patients has been strengthened, the medication adherence of patients has been greatly improved, and their quality of life has been significantly enhanced $(15,16)$. The complete pharmaceutical care team includes doctors, pharmacists, nursing staff, and other paramedical staff. Their collaborative work can resolve DRPs and improve the quality of medical services $(8,17)$.

This is the first study that reports DRPs in nephrology department by clinical pharmacists in China, which proves that the clinical pharmacists play an active role in the drug safety of CKD patients. Through the intervention of clinical pharmacists, potential medication errors and adverse drug reactions can be effectively avoided, and the medication safety of patients can be further improved. Granted, this study has the role of a pioneer to a certain extent. Despite such fact, though, our study has its short-comings. There are several limitations that need to be considered. First, our study was limited to one hospital with a small sample size and no control group. Accordingly, we compared the results with the similar research in other countries. In further studies, the model of pharmacy service should be validated with a larger population and the studies with control group are necessary to be carried out. Apart from that, there are researches in DRPs in more clinical departments such as cardiology, respiratory department, orthopedics, endocrinology, and nephrology, but we lack data of DRPs on the comparison between different departments.

\section{Conclusions}

There is a high rate of DRPs in CKD patients in our hospital. In our study, a total $77 \%$ of the patients had DRPs. Clinical pharmacists can identify DRPs and implement appropriate interventions to prompt rational drug use.

\section{Acknowledgments}

Funding: Jiangsu Pharmaceutical Association - Hengrui Hospital Pharmacy Fund (H202032).

\section{Footnote}

Reporting Checklist: The authors have completed the STROBE reporting checklist. Available at https://dx.doi. org/10.21037/apm-21-817

Data Sharing Statement: Available at https://dx.doi. org/10.21037/apm-21-817

Conflicts of Interest: All authors have completed the ICMJE uniform disclosure form (available at https://dx.doi. org/10.21037/apm-21-817). The authors have no conflicts of interest to declare.

Ethical Statement: The authors are accountable for all aspects of the work in ensuring that questions related to the accuracy or integrity of any part of the work are appropriately investigated and resolved. All procedures performed in this study involving human participants were in accordance with the Declaration of Helsinki (as revised in 2013). The study was approved by the Ethics Committee of the First Affiliated Hospital of Soochow University (NO.: (2021)146) and informed consent was taken from all the 
patients.

Open Access Statement: This is an Open Access article distributed in accordance with the Creative Commons Attribution-NonCommercial-NoDerivs 4.0 International License (CC BY-NC-ND 4.0), which permits the noncommercial replication and distribution of the article with the strict proviso that no changes or edits are made and the original work is properly cited (including links to both the formal publication through the relevant DOI and the license). See: https://creativecommons.org/licenses/by-nc-nd/4.0/.

\section{References}

1. GBD Chronic Kidney Disease Collaboration. Global, regional, and national burden of chronic kidney disease, 1990-2017: a systematic analysis for the Global Burden of Disease Study 2017. Lancet 2020;395:709-33.

2. Foreman KJ, Marquez N, Dolgert A, et al. Forecasting life expectancy, years of life lost, and all-cause and causespecific mortality for 250 causes of death: reference and alternative scenarios for 2016-40 for 195 countries and territories. Lancet 2018;392:2052-90.

3. Zhang L, Wang F, Wang L, et al. Prevalence of chronic kidney disease in China: a cross-sectional survey. Lancet 2012;379:815-22. Erratum in: Lancet 2012;380:650.

4. Zhang L, Zhao MH, Zuo L, et al. China Kidney Disease Network (CK-NET) 2016 Annual Data Report. Kidney Int Suppl (2011) 2020;10:e97-e185.

5. Pharmaceutical Care Network Europe. Classification for drug related problems. Available online: https://www. pcne.org/upload/files/334_PCNE_classification_V9-0.pdf. Accessed 2 Sept 2020.

6. van Mil JW, Westerlund LO, Hersberger KE, et al. Drugrelated problem classification systems. Ann Pharmacother 2004;38:859-67.

7. Basger BJ, Moles RJ, Chen TF. Application of drugrelated problem (DRP) classification systems: a review of the literature. Eur J Clin Pharmacol 2014;70:799-815.

Cite this article as: Liu XX, Wang HX, Hu YY, Zhu XT, Tan $\mathrm{X}$, Yang Y, Hang YF, Zhu JG. Drug-related problems identified by clinical pharmacists in nephrology department of a tertiary hospital in China-a single center study. Ann Palliat Med 2021;10(8):8701-8708. doi: 10.21037/apm-21-817
8. Dvořáčková E, Rychličková J, Pávek P, et al. Analysis and management of drug related problems on a nephrology ward from a pharmacist's point of view. Pharmazie 2019;74:625-9.

9. St Peter WL, Wazny LD, Patel UD. New models of chronic kidney disease care including pharmacists: improving medication reconciliation and medication management. Curr Opin Nephrol Hypertens 2013;22:656-62.

10. Morisky DE, Ang A, Krousel-Wood M, Ward HJ. Predictive validity of a medication adherence measure in an outpatient setting. J Clin Hypertens (Greenwich) 2008; $10: 348-54$.

11. Bayliff CD, Einarson TR. Physician assessment of pharmacists' interventions--a method of estimating cost avoidance and determining quality assurance. Can J Hosp Pharm 1990;43:167-71, 195.

12. Subeesh VK, Abraham R, Satya Sai MV, et al. Evaluation of prescribing practices and drug-related problems in chronic kidney disease patients: A cross-sectional study. Perspect Clin Res 2020;11:70-4.

13. Mason NA. Polypharmacy and medication-related complications in the chronic kidney disease patient. Curr Opin Nephrol Hypertens 2011;20:492-7.

14. van den Bemt PM, Egberts TC, de Jong-van den Berg LT, et al. Drug-related problems in hospitalised patients. Drug Saf 2000;22:321-33.

15. Stemer G, Lemmens-Gruber R. Clinical pharmacy activities in chronic kidney disease and end-stage renal disease patients: a systematic literature review. BMC Nephrol 2011;12:35.

16. Arroyo Monterroza DA, Castro Bolivar JF. Pharmaceutical care practice in patients with chronic kidney disease. Farm Hosp 2017;41:137-49.

17. Belaiche S, Romanet T, Allenet B, et al. Identification of drug-related problems in ambulatory chronic kidney disease patients: a 6-month prospective study. J Nephrol 2012;25:782-8. 